\title{
Ferramenta de apoio à determinação de concentração de PCBs em óleo mineral isolante
}

\author{
Débora C. Marcilio, Ana P. Oening, Daniel H. M. Detzel, Heloisa N. da Motta, \\ Kassia dos Santos, Mariana C. Coelho, André E. Lazzaretti. \\ LACTEC \\ 81531-980, Centro Politécnico da UFPR, Curitiba, PR \\ E-mail: debora@lactec.org.br \\ Erick Amaral Campos. \\ CTEEP \\ E-mail: ericampos@cteep.com.br
}

\begin{abstract}
Resumo: A maioria das concessionárias de energia elétrica possui equipamentos com isolamento à base de óleo mineral, sujeitos à contaminação por compostos do tipo bifenilas policloradas (PCBs), altamente poluentes. No Brasil, as restrições de uso das PCBs foram implementadas pela Portaria Interministerial 19, de 2 de janeiro de 1981, que proibiu a fabricação, comercialização e uso das PCBs em todo território nacional. A identificação e quantificação dessas substâncias em óleo mineral, como é realizado atualmente, é baseada no conhecimento do técnico, o que torna o processo subjetivo. A pesquisa desenvolvida é produto do projeto de P\&D desenvolvido pelo LACTEC em conjunto com a CTEEP - Companhia de Transmissão de Energia Elétrica Paulista e tem como objetivo o desenvolvimento de uma nova metodologia para quantificação do teor de PCBs nas amostras de óleo mineral, baseada em cálculos numéricos, visando reduzir a subjetividade desta quantificação.
\end{abstract}

\section{Introdução}

A maioria das empresas concessionárias de energia elétrica possui equipamentos com isolamento à base de óleo mineral, tais como transformadores de potência, capacitores, entre outros. A contaminação deste líquido isolante por compostos do tipo bifenilas policloradas, conhecidas como PCBs, é uma possibilidade real e que deve ser considerada pelas áreas responsáveis pelos passivos ambientais de cada empresa.

PCBs são substâncias sintéticas enquadradas no âmbito da Convenção de Estocolmo, como um dos 12 Poluentes Orgânicos Persistentes - POPs, que devem ser eliminados por apresentarem riscos ambientais e à saúde humana, sendo classificados como resíduos perigosos, em função da sua toxicidade, no âmbito da Norma NBR 10.004. A sua potencialidade de risco ao meio ambiente e à saúde humana advém de suas características intrínsecas. Devido à sua alta persistência, permanecem presentes por longos períodos no ambiente. Não são biodegradáveis e podem se acumular nos tecidos gordurosos dos seres vivos, sendo também altamente tóxicos, mesmo em baixas concentrações. Apresentam ainda capacidade de percorrer longas distâncias, propagando-se pelo ar, pela água e por espécies migratórias. Podem causar anomalias à saúde humana, como problemas nos sistemas imunológico, cardiovascular, endócrino, gastrointestinal, respiratório e reprodutivo.

No Brasil, as restrições de uso das PCBs foram implementadas pela Portaria Interministerial 19, de 2 de janeiro de 1981, que proibiu a fabricação, comercialização e uso das PCBs em todo território nacional. Porém permitiu que os equipamentos em uso continuassem em funcionamento até sua substituição integral ou até a troca do fluido isolante por outro isento de PCBs. A Instrução Normativa 001 SEMA/STC/CRC, do Ministério do Interior, de 1983 regulamentou as condições que devem ser observadas no manuseio, armazenagem e transporte de PCBs e/ou material contaminado. Desde 2004 o Brasil participa da Convenção de Estocolmo que prevê a eliminação progressiva de PCBs até 2025 de transformadores, capacitores e demais equipamentos elétricos contendo PCBs ou óleos contaminados com PCBs e por seus demais resíduos. As PCBs foram comercializadas em território nacional principalmente com o nome de 
Aroclor ${ }^{\circledR}$, sendo os mais comuns para uso em equipamentos elétricos o Aroclor ${ }^{\circledR} 1242$, o Aroclor ${ }^{\circledR} 1254$ e o Aroclor® 1260 [6].

Atualmente no Brasil a quantificação do teor de PCBs em amostras de óleos isolantes é realizada conforme a norma ABNT NBR 13882 - "Líquidos isolantes elétricos - Determinação do teor de bifenilas policloradas" [1], utilizando a técnica analítica de cromatografia a gás com detector de captura de elétrons. Observações feitas em trabalhos interlaboratoriais têm mostrado que a metodologia e critérios utilizados atualmente, como a comparação visual dos picos do cromatograma da amostra com soluções padrão como critério de diagnóstico, não são precisos o suficiente e permitem que um equipamento possa ser classificado erroneamente como contaminado, implicando em elevados custos para destinação final dos mesmos. Como pode se verificar na Tabela 1, que mostra o resultado de três trabalhos interlaboratoriais de quantificação de PCBs pela norma citada, onde ocorreu uma grande variação na determinação da concentração de PCBs [2], [3] e [4].

\begin{tabular}{|c|c|c|c|c|}
\cline { 3 - 5 } \multicolumn{1}{c|}{} & \multirow{2}{*}{$\mathrm{N}^{\circ}$ laboratórios } & \multicolumn{3}{|c|}{ Concentração $\mathrm{mg} / \mathrm{kg}$} \\
\cline { 3 - 5 } participantes & Valor minimo & Valor máximo & Média \\
\hline nov/09 & 10 & 15,6 & 50 & 40,4 \\
\hline mai/11 & 12 & 7 & 50 & 37 \\
\hline abr/12 & 13 & 5,9 & 42 & 26,9 \\
\hline
\end{tabular}

Tabela 1: Resultados de trabalhos interlaboratoriais - teor de PCBs em óleo mineral isolante.

Com um laudo de análise incorreto, pode-se destinar à incineração um material não contaminado, gerando elevados custos desnecessários, bem como dispor no ambiente material contaminado, causando uma série de prejuízos ambientais difíceis de serem eliminados.

Dessa maneira, o objetivo dessa pesquisa é o desenvolvimento de uma nova metodologia para quantificação do teor de PCBs nas amostras de óleo mineral, determinando assim a melhor maneira de se automatizar o processo realizado pelo técnico.

\section{Metodologia Atual}

Ao longo do tempo de operação do equipamento elétrico, o óleo isolante sofre degradação termo-oxidativa, a qual gera compostos oxidados que eluem da coluna cromatográfica na mesma faixa de tempos de retenção que as PCBs, interferindo significativamente na sua quantificação. Quando mais envelhecido estiver o fluido isolante, maior a presença destes interferentes na amostra a ser analisada. Por este motivo, o processo realizado para quantificar o teor de PCBs nas amostras de óleo mineral é altamente baseado em conhecimentos do técnico. Através de curvas de calibração geradas a partir de padrões dopados com diferentes concentrações de PCBs, o técnico compara, visualmente, as curvas geradas para as amostras e para os padrões e dessa maneira, identifica e exclui do cromatograma os picos que não caracterizam a ocorrência de PCBs, mas sim a oxidação do óleo mineral analisado. $\mathrm{O}$ técnico segue os seguintes passos: Identificação prévia da concentração de PCBs; Identificação e remoção de oxidação e determinação da concentração de PCBs na amostra.

A identificação prévia da concentração tem como finalidade identificar qual(is) Aroclor aparece $(\mathrm{m})$ na amostra e determinar qual curva de calibração será utilizada na quantificação. A identificação de oxidação é uma tarefa difícil de ser realizada, pois a ocorrência da oxidação pode ser confundida com o Aroclor 1242. Se o técnico classificar um pico de oxidação como Aroclor 1242, o valor quantificado terá um valor maior que o real. E para o caso contrário, onde um pico de Aroclor 1242 seja classificado como oxidação, o valor quantificado terá um valor menor. Em ambos os casos tem-se resultados incorretos.

\section{Metodologia Proposta}

A curva oriunda da análise de cromatografia é uma função temporal onde a cada intervalo de tempo tem-se uma quantidade de PCBs. A principal característica dessa curva são picos e vales, ou seja, os valores que compõem o cromatograma têm aumentos e decréscimos 
bruscos, o que não caracteriza uma curva suave. Algumas técnicas aplicadas [10] a funções podem ser utilizadas nos resultados da cromatografia a fim de se analisar o comportamento dos mesmos ou até auxiliar na identificação, classificação e quantificação de PCBs.

A metodologia desenvolvida segue os principais passos que o técnico realiza. Todo processo é baseado em valores de referência, onde são registrados o início, o pico e o fim do intervalo de ocorrência de PCBs.

Primeiramente é pré-determinado se a amostra é composta apenas por óleo oxidado ou contaminado com Aroclor 1242, Aroclor 1254, Aroclor 1260 ou com o mix dos três Aroclors. Os picos de maior intensidade de PCBs são identificados fazendo-se uma varredura no intervalo de vale a vale, onde o pico de referência está compreendido. Esse processo é realizado tanto nos padrões quanto nas amostras a serem analisadas.

Os valores de maior intensidade para cada tipo de padrão são utilizados para o cálculo da média, com isso têm-se cromatogramas médios de padrões. Por exemplo, considere cinco cromatogramas do Aroclor 1242, armazenados no banco de dados. Após a identificação das intensidades máximas para cada cromatograma, é calculada a média entre os picos coincidentes dos diferentes cromatogramas, e assim tem-se um cromatograma médio do padrão Aroclor 1242.

Para o cálculo da correlação, os cromatogramas médios e das amostras passam pela normalização dos dados; utilizando-se como critério a norma pelo mínimo. Para realizar o processo de classificação, optou-se por utilizar o coeficiente de correlação de Pearson [8] e [9]. Esse coeficiente indica o grau e a direção da correlação linear existente entre duas variáveis. No caso dos cromatogramas, indica a correlação existente entre a amostra sob análise e cada uma das referências disponibilizadas: Aroclor 1242, Aroclor 1254, Aroclor 1260, mix e amostra contendo apenas oxidação. Em geral, adota-se o seguinte critério para análise do coeficiente calculado:

- $\rho=1$ : significa uma correlação linear perfeita (e positiva) entre as duas variáveis de entrada;

- $\rho=-1$ : significa uma correlação perfeita (e negativa) entre as duas variáveis de entrada;

- $\quad \rho=0$ : indica que as duas variáveis não possuem dependência linear, uma em relação à outra. No entanto, é importante destacar que pode existir uma dependência não linear entre essas variáveis.

A análise da linha base do cromatograma é uma etapa muito importante, pois a variação temporal do comportamento da linha base pode ser associada a uma componente de baixa frequência presente no cromatograma com oxidação. Ou seja, a presença de oxidação faz com que a linha base deixe de ser constante e próxima de zero, tornando-se oscilatória com frequência de oscilação na ordem de $\mathrm{mHz}$ com maior ocorrência na região do Aroclor 1242 e atenuando-se para os instantes finais do cromatograma. Dentre os testes realizados para o tratamento da linha base optou-se por três metodologias:

- União vale a vale somente dos intervalos que contêm os picos de PCBs: Nesse caso é realizada a união dos intervalos que contêm pico de PCBs. Os pontos que sinalizam o início e o fim dos intervalos são fornecidos previamente, e usando o mesmo princípio da identificação do pico com maior intensidade, é realizada uma investigação nos padrões e nas amostras para selecionar os pontos correspondentes aos pontos de início e fim usados como referência.

- União vale a vale de todos os dados: Nesse caso são identificados os picos e os vales de toda amostra e realizada a união dos vales. A identificação dos vales é feita através da comparação entre os pontos da curva para selecionar os de menor intensidade.

- Interpolação dos dados que pertencem aos intervalos que contêm os picos de PCBs: Aqui os dados referentes aos intervalos em que os picos de PCBs aparecem são interpolados e é traçada uma reta entre eles. Os pontos desse intervalo são subtraídos dessa reta. Assim, a diferença na linha base é eliminada e consegue-se obter uma curva mais regular para ser trabalhada. 
Após o tratamento da linha base, tanto para o caso onde foi realizada a união vale a vale somente dos intervalos que contém os picos de PCBs, quanto para o caso onde foi realizada a união vale a vale de todos os dados, temos duas curvas. Para finalizar o tratamento da linha base é necessário eliminar essa diferença. Para isso foram calculadas as integrais [10] dessas curvas e subtraído os valores; com isso foi obtida a área total de PCBs.

Para o caso onde foi realizada a interpolação [7] dos dados que pertencem aos intervalos que contêm os picos de PCBs, tem-se uma única curva para se calcular a área, pois quando foi efetuada a subtração entre os dados do cromatógrafo e a reta já foram retirados os resíduos.

São calculadas as áreas [7] dos padrões e das amostras, sendo que a área do padrão é usada para calcular os coeficientes da equação da reta que servirá para quantificar as amostras (curva de calibração). Para a determinação da reta que será usada na quantificação de PCBs é necessário padrões com diferentes concentrações. Com as metodologias de tratamento da linha base, têm-se três áreas calculadas para cada padrão e amostra, sendo assim haverá três resultados de quantificação para cada amostra.

Por fim, o valor das concentrações é fornecido em forma de intervalo, ou seja, através do cálculo da média e do desvio padrão [5] entre os três resultados para cada amostra resultando em um limite inferior (desvio padrão subtraído da média) e um limite superior (desvio padrão acrescido a média), que determinam o intervalo de confiança para a concentração de PCBs da amostra.

\section{Testes e Resultados}

Para os testes realizados, com a finalidade de validar a metodologia proposta, foram comparadas as quantificações encontradas pela metodologia e as realizadas pelo técnico. Nesse artigo, são apresentados resultados de nove amostras de validação, preparadas com óleo mineral isolante com diferentes níveis de oxidação, dopado com diferentes concentrações de Aroclors.

A Tabela 2 contém a composição de cada amostra, por exemplo, a amostra 1 é um óleo mineral (OMI) novo dopado com $50 \mathrm{mg} / \mathrm{L}$ dos três tipos de Aroclors. A Tabela 3 contêm os valores de concentrações reais e os encontrados pelo técnico, onde pode ser observada uma diferença entre os valores obtidos. Isso ocorre porque o florisil, material utilizado no prétratamento das amostras com o objetivo de eliminar os interferentes da matriz (OMI) como, por exemplo, os compostos polares provenientes da oxidação do OMI acabam retendo também parte do analito (PCBs).

\begin{tabular}{|c|c|}
\hline Amostras & Descrição \\
\hline 1 & OMI novo com 50mg/L de PCBs \\
\hline 2 & OMI novo com $85 \mathrm{mg} / \mathrm{L}$ de PCBs \\
\hline 3 & OMI novo com $15 \mathrm{mg} / \mathrm{L}$ de PCBs \\
\hline 4 & OMl pouco oxidado com $85 \mathrm{mg} / \mathrm{L}$ de PCBs \\
\hline 5 & OMl pouco oxidado com $50 \mathrm{mg} / \mathrm{L}$ de $\mathrm{PCBs}$ \\
\hline 6 & OMI pouco oxidado com 15mg/L de PCBs \\
\hline 7 & OMI muito oxidado com $85 \mathrm{mg} / \mathrm{L}$ de PCBs \\
\hline 8 & OMI muito oxidado com $15 \mathrm{mg} / \mathrm{L}$ de PCBs \\
\hline 9 & OMI muito oxidado com $50 \mathrm{mg} / \mathrm{L}$ de $\mathrm{PCBs}$ \\
\hline
\end{tabular}

Tabela 2: Composição das amostras.

\begin{tabular}{|c|c|c|}
\cline { 2 - 3 } \multicolumn{1}{c|}{} & \multicolumn{2}{c|}{ concentração mg/L } \\
\hline Amostras & real & encontrada \\
\hline 1 & 50 & 42,70 \\
\hline 2 & 85 & 84,60 \\
\hline 3 & 15 & 13,20 \\
\hline 4 & 85 & 76,90 \\
\hline 5 & 50 & 42,20 \\
\hline 6 & 15 & 13,30 \\
\hline 7 & 85 & 82,70 \\
\hline 8 & 15 & 18,00 \\
\hline 9 & 50 & 47,90 \\
\hline
\end{tabular}

Tabela 3: Concentração real e encontrada pelo técnico. 
Primeiramente, foi realizada a pré-classificação das amostras - esse resultado está descrito na Tabela 4. Segundo esses resultados, a amostra 1, por exemplo, tem uma maior correlação com o mix dos tipos de Aroclors.

Quando analisadas as correlações com os padrões de oxidação percebe-se que o método apresentou resultados satisfatórios, pois nas três primeiras amostras as correlações com a oxidação foram baixas e as três últimas apresentaram correlações maiores, o que confere com as características fornecidas na Tabela 2.

\begin{tabular}{|c|c|c|c|c|c|}
\cline { 2 - 6 } \multicolumn{1}{c|}{} & \multicolumn{5}{|c|}{ Classificação } \\
\hline Amostras & Aroclor 1242 & Aroclor 1254 & Aroclor 1260 & mix & Oxi \\
\hline 1 & $-0,07$ & 0,06 & 0,06 & 0,95 & $-0,03$ \\
\hline 2 & $-0,08$ & 0,07 & 0,09 & 0,93 & $-0,04$ \\
\hline 3 & $-0,05$ & 0,10 & 0,10 & 0,93 & $-0,01$ \\
\hline 4 & $-0,01$ & 0,07 & 0,03 & 0,55 & 0,01 \\
\hline 5 & $-0,01$ & 0,08 & 0,03 & 0,55 & 0,02 \\
\hline 6 & 0,11 & 0,08 & 0,01 & 0,55 & 0,14 \\
\hline 7 & 0,12 & 0,00 & $-0,01$ & 0,75 & 0,19 \\
\hline 8 & 0,31 & $-0,17$ & $-0,16$ & 0,12 & 0,42 \\
\hline 9 & 0,25 & $-0,07$ & $-0,09$ & 0,49 & 0,34 \\
\hline
\end{tabular}

Tabela 4: Correlação entre as amostras e os padrões.

Avaliando as correlações obtidas, o técnico poderá decidir se acata a sugestão de padrão para quantificar as amostras ou se adota o mix dos Aroclors para realizar a tarefa. A metodologia desenvolvida prevê essa interferência, utilizando desta forma os conhecimentos do técnico.

\begin{tabular}{|c|c|c|c|}
\hline Amostras & Limite Superior & Pico & Limite Inferior \\
\hline 1 & 40 & 41 & 42 \\
\hline 2 & 82 & 83 & 84 \\
\hline 3 & 14 & 14 & 14 \\
\hline 4 & 58 & 63 & 69 \\
\hline 5 & 35 & 37 & 39 \\
\hline 6 & 13 & 13 & 14 \\
\hline 7 & 93 & 98 & 102 \\
\hline 8 & 43 & 46 & 48 \\
\hline 9 & 70 & 71 & 73 \\
\hline
\end{tabular}

Tabela 5: Quantificação pela metodologia.

Analisando-se os resultados encontrados na metodologia, Tabela 5, e pelo técnico, Tabela 3, nota-se que para as primeiras amostras a metodologia conseguiu resultados próximos dos encontrados pelo técnico, levando em consideração a influência do florisil.

Porém para as últimas amostras, que foram preparadas com óleo mineral muito oxidado, ocorreu uma distorção nos resultados, confirmando o fato da oxidação realmente interferir na quantificação.

Os testes realizados mostraram que a nova metodologia desenvolvida pode ser utilizada pelo técnico como uma ferramenta auxiliar para quantificar as amostras, pois a metodologia poderá ajudá-lo nas tomada de decisões. Com isso, o técnico contará também com um embasamento cientifico e não somente com a subjetividade.

\section{Conclusão}

A norma Brasileira vigente é incipiente quanto à determinação do teor de PCBs em óleos isolantes, sendo o processo de quantificação, conforme realizado, altamente dependente dos conhecimentos do técnico e com isso as análises realizadas para determinação da concentração de PCBs em líquidos isolantes se tornam subjetivas. A metodologia elaborada nessa pesquisa, por sua vez, é composta por conceitos usados pelo técnico e também cálculos numéricos, reduzindo a subjetividade da quantificação realizada.

A validação da metodologia com amostras reais, de concentração desconhecida, mostrou-se uma tarefa difícil de ser realizada, pois há apenas a avaliação do técnico para ser 
tomada como referência. Assim optou-se por utilizar amostras onde as condições são conhecidas (amostras de validação). Comparando-se os resultados das quantificações efetuadas pela metodologia desenvolvida com os resultados do técnico, a nova metodologia apresentou resultados satisfatórios.

\section{Referências}

[1] ASSOCIAÇÃO BRASILEIRA DE NORMAS TÉCNICAS. NBR 13882: Líquidos isolantes elétricos - Determinação do teor de bifenilas policloradas (PCB). Rio de Janeiro, 2008.

[2] COMITÊ DE ESTUDOS DE MATERIAIS E TECNOLOGIAS EMERGENTES - CE D1. $5^{\text {a }}$ Circulação de amostras de óleo mineral isolante para comparação interlaboratorial de resultados de ensaios físico-químicos e cromatográficos, 2009, p. 50-54. Relatório Técnico (Relatório Técnico D1.01 - 002/2009). Grupo de trabalho D1.01 - Líquidos Isolantes, CIGRÉ BRASIL, Rio de Janeiro.

[3] COMITÊ DE ESTUdOS DE MATERIAIS E TECNOLOGIAS EMERGENTES - CE D1. $7^{\text {a }}$ Circulação de amostras de óleo mineral isolante para comparação interlaboratorial de resultados de ensaios físico-químicos e cromatográficos, 2010, p. 139-143. Relatório Técnico (Relatório Técnico D1.01 - 002/2010). Grupo de trabalho D1.01 - Líquidos Isolantes, CIGRÉ BRASIL, Rio de Janeiro.

[4] COMISSÃO DE ESTUDOS DE MATERIAIS E TECNOLOGIAS EMERGENTES - CE D1. $8^{\text {a }}$ Circulação de amostras de óleo mineral isolante para comparação interlaboratorial de resultados de ensaios físico-químicos e cromatográficos, 2012, p. 115-118. Relatório Técnico (Relatório Técnico D1.01 - 001/2012). Grupo de trabalho D1.01 - Líquidos Isolantes, CIGRÉ BRASIL, Rio de Janeiro

[5] Ferreira, D. F. Estatística Multivariada. Lavras: Ed. UFLA, pp. 662, 2008.

[6] Penteado, J. C. P.; Vaz J.M.; O legado das Bifelinas Policloradas (PCBs), Quim. Nova, Vol 24, No. 3, pp. 390-398, 2001.

[7] Ruggiero, M. A.G.; Lopes, V. L. R. Cálculo Numérico - Aspectos Teóricos e Computacionais, 1996, MAKRON Books.

[8] Theodoridis, S.; Koutroumbas, K. Pattern Recognition, 2009, 4 a Edição, Academic Press.

[9] Theodoridis, S.; Pikrakis, A.; Koutroumbas, K.; Cavouras, D. Introduction to Pattern Recognition - A MATLAB Approach, 2010, Academic Press.

[10] Stewart, J. Cálculo, volume 1, McMaster University e University of Toronto, 2013. 